INRA Prod. Anim., 2017, 30 (4), 273-284

\title{
La « grange » : un cadre conceptuel pour appréhender les bouquets de services rendus par l'élevage dans les territoires
}

\author{
M. DURU ${ }^{1}$, C. DONNARS 2 , J. RYCHAWY', O. THEROND 3 , B. DUMONT \\ ${ }^{\prime}$ UMR AGIR, INRA, Université Toulouse, INPT, 31326, Castanet Tolosan, France \\ 2 DEPE, INRA, 147 rue de l'Université, 75338, Paris, France \\ ${ }_{3}^{3}$ UMR LAE, INRA, Université de Lorraine, 68021, Colmar, France \\ ${ }^{4}$ Université Clermont Auvergne, INRA, Vetagro Sup, UMR Herbivores, 63122, Saint-Genès-Champanelle, France \\ Courriel:michel.duru@inra.fr
}

Le rapport «Livestock's long shadow » de la FAO a fortement orienté les débats en mettant en balance les enjeux de sécurité alimentaire et les dommages climatiques et environnementaux associés à l'élevage. Certaines thématiques ont depuis été précisées (niveau d'émissions de gaz à effet de serre par les animaux, potentiel de séquestration du carbone des prairies) ou ont émergé (concept de santé globale), mais les enjeux restent encore souvent examinés de manière sectorielle. Cet article propose un cadre d'analyse pour représenter de manière structurée la diversité des services et impacts issus de l'élevage dans les territoires.

Le rapport « Livestock's long shadow》 s'inscrit dans la lignée des études qui interrogent les conséquences d'une croissance de la population mondiale jusqu'à 9,7 milliards d'habitants en 2050 et d'un accroissement de la part des protéines animales dans les régimes alimentaires. En recensant les impacts environnementaux de l'élevage sur l'usage des terres ${ }^{1}, l^{\prime}$ 'eau, la biodiversité et sa contribution au changement climatique, ce rapport a alerté sur la menace pour l'avenir - «l'ombre portée »-que représenterait son développement, et proposé quelques pistes pour y remédier. Un chiffre a surtout été retenu : les animaux d'élevage seraient à l'origine de $14,5 \%$ des émissions totales de gaz à effet de serre (Gerber et al 2013). Selon ce rapport, les émissions sont pour plus des 3/4 liées aux ruminants et pour un peu moins d'un 1/4 aux monogastriques. Elles ont trois grandes origines : les changements d'usage des terres liés à l'alimentation du bétail (dont la déforestation), les émissions issues des effluents d'élevage et le méthane éructé par les ruminants. Ce rapport pointe également les perturbations que l'élevage intensif induit dans les cycles bio-géochimiques (Rockström et al 2009). Ainsi, le commerce international conduit à fortement déconnecter les sites de production des lieux de consommation. Les impacts environnementaux de l'élevage sont ainsi souvent difficiles à tracer, et varient selon l'origine des productions (Billen et al 2015, Chaudhary et Kastner 2016). Le rapport rappelle toutefois que lorsque l'alimentation est basée sur des prairies, ces impacts négatifs sont réduits du fait du stockage de carbone dans les sols et de la présence d'une biodiversité importante.

Par ailleurs, la compétition entre 1'alimentation animale et l'alimentation humaine est un problème pour la sécurité alimentaire mondiale ; les analyses pointent la faible efficacité de conversion énergétique et protéique des productions végétales par les ruminants, et la concurrence des monogastriques pour les céréales (Tilman et Clark 2014). Selon Pimentel (2003), il faudrait en moyenne $6 \mathrm{~kg}$ de protéines végétales pour fabriquer $1 \mathrm{~kg}$ de protéines animales avec de fortes variations selon les systèmes de production; les monogastriques sont en général plus efficients pour la transformation des protéines végétales en protéines animales, alors les ruminants peuvent utiliser des prairies et parcours impropres à la culture ce qui réduit la concurrence avec l'alimentation humaine. Certains calculs indiquent que les ruminants pourraient ainsi syn- thétiser plus de protéines qu'ils ne consomment de protéines directement utilisables pour l'alimentation humaine (Peyraud 2017). En lien avec ces préoccupations, différents travaux analysent le potentiel de recyclage des coproduits industriels et des déchets du système agro-alimentaire dans l'alimentation des animaux (van Zanten et al 2016).

Aux préoccupations environnementales ou liées à la sécurité alimentaire mondiale s'ajoutent celles associées à une consommation importante de protéines animales vis-à-vis de maladies chroniques (cancers, maladies cardiovasculaires, obésité) ; elles sont aujourd'hui identifiées comme un enjeu majeur de santé publique. Ainsi, les recommandations récentes de l'Anses (Anses 2016) préconisent-elles une réduction de la consommation de protéines animales, en particulier de viandes rouges, charcuteries et de produits transformés, et pointent l'intérêt de consommer des produits riches en acide alpha-linolénique dont nous sommes déficitaires. Les protéines animales et végétales ne présentent cependant pas les mêmes équilibres en acides aminés essentiels et ne sont donc pas entièrement substituables (Chardigny et Walrand 2016). 
Les approches systémiques les plus classiques évaluant les services et impacts de l'élevage et des produits animaux portent sur les flux marchands, et les flux de matières et de nutriments. Elles reposent le plus souvent sur des données à une échelle large qui ne prennent pas en compte la diversité des systèmes au sein des territoires. Les analyses en cycle de vie appliquées aux territoires permettent de visualiser les transferts successifs de la matière depuis sa fabrication jusqu'à sa consommation et au recyclage des déchets alimentaires. Ces approches se focalisent sur l'efficience d'utilisation des ressources, en identifient les sources et quantifient les pertes vers l'atmosphère, l'eau et les sols. La communauté scientifique spécialiste de l'azote est particulièrement active dans ce domaine, appliquant à un territoire le principe de bilan des flux de nutriments entrants et sortants. Billen et al (2015) ont ainsi quantifié l'importance des échanges lointains de nutriments et de matières. Westhoek et al (2015) ont simulé les effets d'une baisse de la consommation de viande de $50 \%$ sur les flux protéiques et ont cartographié ses effets sur les excédents d'azote à l'échelle européenne. À ce jour, la plupart des approches systémiques excluent encore les effets de la diversité des régimes alimentaires et leurs dimensions culturelles (par exemple l'intérêt croissant pour le végétarisme en réponse à l'indignité de certaines conditions d'élevage et d'abattage). Cependant, les approches de métabolisme territorial, et plus récemment le concept de nutrition environnementale (Sabate et al 2016), permettent de relier les processus naturels aux caractéristiques sociales et techniques d'un territoire.

L'ensemble de ces questions met en exergue la nécessité d'identifier plus précisément les services et impacts de l'élevage et de ses produits dans les domaines biophysique et socioéconomique. Dans la première partie de cet article nous pro- posons une grille d'évaluation multicritères pour répondre à cet enjeu. Nous en dérivons une représentation structurée et simplifiée des services et impacts rendus par l'élevage dans un territoire. Enfin, nous abordons la question de la diversité des systèmes d'élevage afin d'analyser dans quelle mesure certaines de ses formes sont mieux à même de répondre aux enjeux déjà mentionnés.

\section{1 / Identifier la diversité des services et impacts de l'élevage et des produits animaux}

L'analyse de la multifonctionnalité des systèmes de production vise à spécifier la pluralité des rôles de l'élevage, en examinant 1'ensemble de ses finalités pour la société. Le concept de multifonctionnalité est apparu sur la scène internationale en 1992, lors du sommet de Rio, conjointement à celui de développe-

Tableau 1. Domaines et critères à considérer pour évaluer les impacts positifs et négatifs de l'élevage.

\begin{tabular}{|c|c|c|c|c|}
\hline \multirow{2}{*}{\multicolumn{2}{|c|}{ Domaines }} & \multirow{2}{*}{ Sous domaines } & \multicolumn{2}{|c|}{ Conditions pour services et impacts } \\
\hline & & & Positifs si & Négatifs si \\
\hline \multirow{5}{*}{ 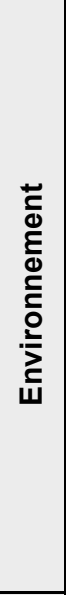 } & \multirow{2}{*}{$\begin{array}{l}\text { Flux de } \\
\text { matières et } \\
\text { d'énergie }\end{array}$} & $\begin{array}{l}\text { Matière } \\
\text { et énergie }\end{array}$ & $\begin{array}{l}\text { - Valorisation des surfaces toujours } \\
\text { en herbe et des co-produits } \\
\text { - Limitation de l'érosion (prairies)* }\end{array}$ & $\begin{array}{l}\text { - Consommation d'intrants exogènes, } \\
\text { pressions locales et exportées sur les } \\
\text { milieux }\end{array}$ \\
\hline & & $\begin{array}{l}\text { Cycles } \\
\text { biogéochimiques }\end{array}$ & $\begin{array}{l}\text { - Prairies (avec légumineuses), } \\
\text { valorisation des matières } \\
\text { organiques, pour la fertilité des sols } \\
\text { et la qualité de l'eau* }\end{array}$ & $\begin{array}{l}\text { - Emissions nettes de gaz à effet de serre } \\
\text { (déstockage de carbone) }\end{array}$ \\
\hline & \multicolumn{2}{|c|}{ Changement climatique } & - Stockage C (prairies) ${ }^{*}$ & - Emissions de GES \\
\hline & \multirow{2}{*}{$\begin{array}{l}\text { Usage des } \\
\text { terres }\end{array}$} & Biodiversité & $\begin{array}{l}\text { - Richesse spécifique dans les } \\
\text { prairies et parcours * }\end{array}$ & $\begin{array}{l}\text { - Faible biodiversité domestique, perte } \\
\text { et sélection de la biodiversité sauvage }\end{array}$ \\
\hline & & $\begin{array}{l}\text { Habitats } \\
\text { et milieux }\end{array}$ & $\begin{array}{l}\text { - Régulations biologiques permises } \\
\text { par les prairies dans les paysages } \\
\text { et les successions de cultures* }\end{array}$ & $\begin{array}{l}\text { - Intensification, conflits avec faune } \\
\text { sauvage }\end{array}$ \\
\hline \multirow{2}{*}{\multicolumn{2}{|c|}{$\begin{array}{l}\text { Socio- } \\
\text { économique }\end{array}$}} & Emploi & $\begin{array}{l}\text { - Création de richesse et d'emplois, } \\
\text { compétences professionnelles } \\
\text { notamment bouchères, } \\
\text { charcutières et fromagères }\end{array}$ & $\begin{array}{l}\text { - Conditions de travail difficiles } \\
\text { dans les élevages, les abattoirs } \\
\text { - Faibles rémunérations des éleveurs }\end{array}$ \\
\hline & & $\begin{array}{l}\text { Valeurs, } \\
\text { patrimoine }\end{array}$ & $\begin{array}{l}\text { - Gastronomie, savoir-faire, } \\
\text { paysages, tourisme, etc. }\end{array}$ & $\begin{array}{l}\text { - Mise en cause des systèmes intensifs, } \\
\text { souffrance animale }\end{array}$ \\
\hline \multirow{2}{*}{\multicolumn{2}{|c|}{$\begin{array}{l}\text { Santé animale } \\
\text { et humaine }\end{array}$}} & $\begin{array}{l}\text { Composition } \\
\text { nutritionnelle et } \\
\text { consommation }\end{array}$ & $\begin{array}{l}\text { - Protéines de qualité, vitamines A, } \\
\text { B12..., oligoéléments, oméga-3 } \\
\text { - Denrées diversifiées }\end{array}$ & $\begin{array}{l}\text { - Teneurs excessives en acides gras } \\
\text { saturés et oméga- } 6 \\
\text { - Excès de consommation de viande } \\
\text { - Antibiorésistance, contamination } \\
\text { médicamenteuse et biocides } \\
\text { des produits animaux et du sol }\end{array}$ \\
\hline & & Santé animale & $\begin{array}{l}\text { - Alimentation équilibrée et intérêt } \\
\text { de plantes à valeur santé* } \\
\text { - Conditions d'élevage et bien-être }\end{array}$ & $\begin{array}{l}\text { - Zoonoses, coûts en santé animale } \\
\text { et humaine } \\
\text { - Pertes de production }\end{array}$ \\
\hline
\end{tabular}

* Services écosystémiques associés (cf. encadré 2). Les références bibliographiques supportant les arguments sont présentées dans le texte et les encadrés 1 et 2. 
Encadré 1. Agriculture, élevage et services écosystémiques.

L'application du cadre conceptuel des services écosystémiques pour analyser les effets et le fonctionnement de l'agriculture conduit à préciser et adapter la classification établie par le Millénium Ecosystem Assessment en 2005 dans laquelle étaient considérés les services d'approvisionnement, de support, de régulation, et les services culturels. II est en effet nécessaire de distinguer les services fournis par les écosystèmes à l'agriculture de ceux fournis à la société (Zhang et al 2007); les premiers sont dénommés « services intrants " (Le Roux et al 2008). Comme proposé par Daily (1997), il est également important de distinguer les services écosystémiques et les biens issus des écosystèmes. En effet, dans les écosystèmes agricoles, la production de biens agricoles (végétaux et animaux) résulte des effets combinés d'intrants industriels exogènes et de services écosystémiques (Duru et al 2015a). Dans les systèmes agroécologiques, une part importante de la production de biens agricoles est fournie par les services écosystémiques.

Pour les productions fourragères, les associations de cultures, les légumineuses, et les rotations longues permettent de réduire l'utilisation d'engrais de synthèse et de pesticides (Kremen et al 2012, Bommarco et al 2013, Duru et al 2015a). Pour les productions animales, l'utilisation de ressources fourragères riches en protéines peut aussi être considérée comme un service intrant car elle contribue à l'autonomie alimentaire. De même, les conditions d'élevage qui permettent de réduire les stress biotiques et les maladies, par exemple par un plus fort lien au sol contribuent au bien-être animal et peuvent même améliorer certains critères de performances (van der Linden et al 2015).

Le développement de services intrants a souvent un effet positif sur les services rendus à la société dans la mesure où les pratiques mises en œuvre sont également susceptibles de réguler le climat (séquestration du carbone par les prairies permanentes et les arbres), d'améliorer la qualité de l'eau, la santé humaine via la qualité des produits (moins de résidus de pesticides ou d'antibiotiques, meilleure composition en acides gras des produits animaux).

ment durable. La multifonctionnalité conduit à considérer l'ensemble des fonctions liées à l'activité agricole audelà de la seule production de biens alimentaires. En effet, l'élevage génère des impacts négatifs sur l'environnement du fait de la consommation d'intrants et des émissions de gaz à effet de serre (tableau 1), mais fournit aussi des services écosystémiques (encadré 1) essentiellement liés aux prairies et à la diversification des systèmes de cultures (encadré 2).

Ryschawy et al (2015) ont recensé quatre catégories de services fournis par l'élevage dans les territoires : 1'approvisionnement (ici la quantité et la qualité de produits animaux), la qualité environnementale (biodiversité, cycles biogéochimiques, diversité des paysages), la vitalité territoriale (dynamisme rural et emploi) et l'identité culturelle (gastronomie, identité des terroirs). Sont ainsi considérées comme services toutes les contributions positives que l'élevage fournit à la société, qui incluent les services écosystémiques, les externalités positives de l'agriculture et la fourniture de biens marchands (Gómez-Baggethun et al 2010). Ceci traduit qu'au-delà de son rôle socio-économique en termes de valeur ajoutée et d'emploi, l'élevage rend aussi des services de nature sociale et patrimoniale. En outre, les produits animaux issus d'une alimentation à l'herbe présentent un intérêt pour la santé humaine (encadré 2). Cependant, les domaines de la santé animale et humaine (tableau 1), étant hors du périmètre de l'expertise scientifique collective sur les rôles, impacts et services issus des élevages en Europe (Dumont et al 2017a), ils n'ont pas été considérés dans l'analyse qui suit. Bien que ces approches systémiques

Encadré 2. Les prairies, principales sources des services écosystémiques fournis par l'élevage.

Les prairies utilisées pour la production de fourrages se retrouvent dans une large gamme de niveaux d'anthropisation (végétations « naturelles » ou semées), de milieux (plaine, montagne, zones sèches ou humides, zones labourables ou non), de degré de pérennisation (prairies permanentes ou temporaires dans les rotations avec des cultures). Les herbacées peuvent y être associées à des espèces ligneuses de manière intentionnelle (pré-vergers, haies, arbres isolés) ou non (parcours, landes). Les prairies fournissent des ressources variées pour l'alimentation animale. L'exploitation de cette diversité au pâturage permet d'augmenter les performances animales (Feng et al 2016). La diversité des ressources peut stimuler l'ingestion des animaux ou réduire les risques de maladies lorsque les animaux consomment des plantes bioactives, par exemple riches en tannins condensés qui favorisent la régulation des parasites des animaux ; les arbres au-delà de leurs fonctions d'ombrage contribuent à l'alimentation des animaux lors des périodes de sécheresse. La mixité des espèces animales au pâturage (d'Alexis et al 2014) et la diversité des types d'animaux au sein d'un troupeau (Ollion et al 2016) constituent d'autres leviers pour valoriser les ressources de par la complémentarité des choix alimentaires des animaux et la variabilité de leur capacité d'ingestion.

Les prairies permanentes stockent du carbone en quantité plus importante que les grandes cultures. En tant que composante d'une mosaïque paysagère, elles contribuent aux contrôles biologiques naturels (régulation des ravageurs, pollinisation) et aux flux de matière et d'éléments nutritifs dans les bassins hydrographiques (services liés à l'eau). La répartition temporelle des prairies dans une rotation de cultures détermine le niveau de séquestration du Carbone, ainsi que la stabilisation et la fertilité du sol. La composition des prairies et leur distribution dans l'espace contribuent à la valeur esthétique des paysages dont on considère qu'elle augmente lorsque la part de prairies fleuries et l'hétérogénéité du paysage augmentent (Lindemann-Matthies et al 2010). Enfin, les rations à base d'herbe étant plus riches en oméga-3 que les rations à base de céréales, les prairies améliorent la valeur nutritionnelle des produits animaux (Pighin et al 2016).

couvrent plusieurs catégories d'impacts, elles présentent cependant des limites. Elles mettent en effet souvent plus l'accent sur les dimensions positives des activités d'élevage que sur leurs impacts environnementaux ou sociaux négatifs, et ce alors même que l'élevage est pointé du doigt vis-à-vis des conditions de travail des éleveurs, de sa faible rémunération, et de la souffrance animale générée par certaines pratiques (tableau 1). Par ailleurs, elles n'évaluent que partiellement les services écosystémiques associés à l'élevage. 
Ainsi, considéré globalement, l'élevage et les produits animaux semblent-ils avoir un rôle ambivalent dans chacun des grands domaines considérés (tableau 1). Il est cependant difficile d'atteindre un haut niveau de services (ou de réduction des impacts) simultanément dans tous les domaines, d'où la nécessité d'évaluer des bouquets de services, i.e. des cooccurrences spatiales de niveaux de services. Pour un système donné, lorsque l'augmentation de la valeur d'un critère induit la diminution de la valeur d'un autre on parle d'antagonismes. À l'inverse, certaines innovations permettent de réduire simultanément plusieurs impacts négatifs, voire de développer des synergies lorsque l'effet combiné des pratiques est supérieur à la somme de leurs effets individuels.

Toutes les formes d'élevage ne se valent pas en termes de niveaux de services et d'impacts. Un enjeu méthodologique est donc de procéder à des évaluations multicritères si possible à différents niveaux d'organisation fonctionnelle : les exploitations d'élevage, les territoires, les filières. Caractériser la nature et le niveau des services et impacts négatifs de l'élevage et des produits animaux reste cependant difficile car ils sont la résultante de processus complexes et d'effets en cascade (Duru et Therond 2015). Janzen (2011) résume le défi méthodologique en insistant sur la nécessité de prendre en compte les pas de temps longs compte tenu des effets différés, les effets délocalisés dans l'espace, la dimension humaine, mais aussi le caractère situé des pratiques, soit l'importance de raisonner les systèmes en fonction de leur contexte local.

Il n'existe pas à notre connaissance de cadre d'analyse permettant d'évaluer les systèmes avec un tel niveau d'intégration. Aussi, dans la partie suivante nous proposons une représentation systémique de l'élevage dans les territoires en veillant à ce que ce cadre conceptuel permette d'appréhender :

i) Les impacts locaux et délocalisés des systèmes d'élevage sur l'environnement et la fourniture de services écosystémiques. Cela suppose d'être à même d'identifier les facteurs clefs permettant de réduire ces impacts, mais aussi de rendre compte des services à l'agriculture et à la société ;

ii) Les services et impacts dans le domaine socio-économique. Cela suppose que les façons de produire, de commercialiser et de consommer soient considérées au-delà de l'insertion des filières dans les marchés et des emplois générés. C'est pourquoi nous considérerons les facteurs déterminant l'acceptation sociale de l'élevage par les citoyens.

\section{2 / Représenter les multiples effets de l'élevage et des produits animaux dans les territoires et les systèmes alimentaires}

Afin de construire une représentation intégrée et structurée de l'élevage et de son insertion dans les territoires et les systèmes alimentaires, nous nous sommes inspirés des recherches sur les systèmes socio-écologiques.

\section{1 / L'élevage et les produits animaux comme un système socio-écologique}

Le concept de « système socio-écologique » permet d'examiner les interactions entre un système social composé d'usagers, individuels et collectifs, mobilisant des technologies et des infrastructures pour gérer des ressources, et un système écologique générant ces ressources. Il a d'abord servi à analyser la complexité des interactions sociales et écologiques de territoires à forts enjeux naturels dans lesquels le système écologique fournit une gamme de services écosystémiques. Les relations entre les fonctions opérant au sein des écosystèmes, les services écosystémiques, les avantages dérivés de ces services, les bénéficiaires de ces services et les valeurs qu'ils attribuent à ces services se traduit par une cascade (Haines-Young et al 2012) qui permet d'identifier comment chaque porteur d'enjeux est influencé ou influence ses différents maillons, des services aux bénéficiaires. Ce type de représentation a été révisé pour prendre en compte les interactions entre les compartiments biologiques et sociaux dans un territoire fortement anthropisé. De leur côté, McGinnis et Ostrom (2014) ont proposé une évolution du cadre conceptuel des systèmes socio-écologiques mettant en lumière l'importance des « situations d'action ». L'enjeu ici est d'examiner comment les caractéristiques sociales et écologiques au sein d'un territoire déterminent les actions des différents acteurs et in fine l'atteinte de différents objectifs et performances à 1'échelle individuelle et collective. Marshall (2015) a formalisé le rôle des systèmes de transformation des biens issus des écosystèmes en introduisant un compartiment « système technique et technologique » à l'interface entre systèmes écologique et social pour traiter des situations où les technologies sont largement déterminantes du mode d'exploitation des ressources naturelles (McGinnis et Ostrom 2014) et des systèmes alimentaires .

Les systèmes d'élevage peuvent ainsi être représentés comme l'articulation entre des systèmes techniques, écologiques et sociaux. La composante biotechnique des systèmes d'élevage correspond aux animaux (pouvant correspondre à plusieurs ateliers conduits séparément ou de manière intégrée par exemple pour l'alimentation), aux cultures fourragères ou non et aux prairies. Les interactions entre ces trois composantes déterminent le niveau de fourniture des services via les flux de matières ou d'énergie, et par conséquent le niveau d'autonomie alimentaire de l'élevage ainsi que les impacts de l'élevage sur l'environnement. La diversité de ces interactions a été décrite et analysée par Moraine et al (2014, 2016). Ces auteurs ont représenté l'élevage sous la forme de trois "sphères », animaux (ruminants et/ou monogastriques), cultures et prairies en interactions dans le temps et l'espace. Une telle représentation permet d'analyser le métabolisme du système et notamment ses impacts sur l'environnement en fonction de la circulation de l'énergie et des matières (intrants, nutriments, aliments, « déchets » et leurs réutilisation) entre les trois sphères. Elle permet aussi de mettre en avant le rôle particulier des prairies, dans la fourniture des services écosystémiques.

Plus largement, les systèmes d'élevage peuvent aussi être vus comme insérés dans des systèmes alimentaires définis comme l'ensemble des technologies et pratiques permettant de produire, traiter, conditionner, distribuer, vendre et consommer des aliments (pentagone de la figure 1). La caractérisation des systèmes alimentaires permet une analyse intégrée des différentes façons de produire et transformer (Foran et al 2014). En complément de la représentation proposée par Moraine et al (2014), celle-ci permet d'illustrer les interactions avec les secteurs amont et aval (figure 1). Les activités en amont du système de production portent sur la fabrication des aliments du bétail et l'agrofourniture ; celles en aval sont l'abattage et la transformation des produits. Dans un territoire d'élevage donné, les dispositifs d'approvisionnement en intrants, de collecte et de transformation des produits, peuvent être internes ou externes au territoire considéré.

Le système écologique correspond aux ressources biotiques (organismes vivants permettant la fourniture des services écosystémiques) et abiotiques (eau, énergie), renouvelables ou non. Les infrastructures/entités spatiales qui conditionnent et permettent le fonctionnement des systèmes agricoles et agroalimentaires peuvent se caractériser à différents niveaux d'organisation (ferme, bassin versant, région, pays, monde), certaines étant inclues dans ou recoupant le territoire analysé (figure 1). Les interactions entre 
Figure 1. Représentation de l'élevage et des produits animaux comme une composante d'un système technique (les filières dans des territoires) en interaction avec un système écologique et un système social (adapté de Marshal 2015 et Vallejo-Rojas et al 2015).

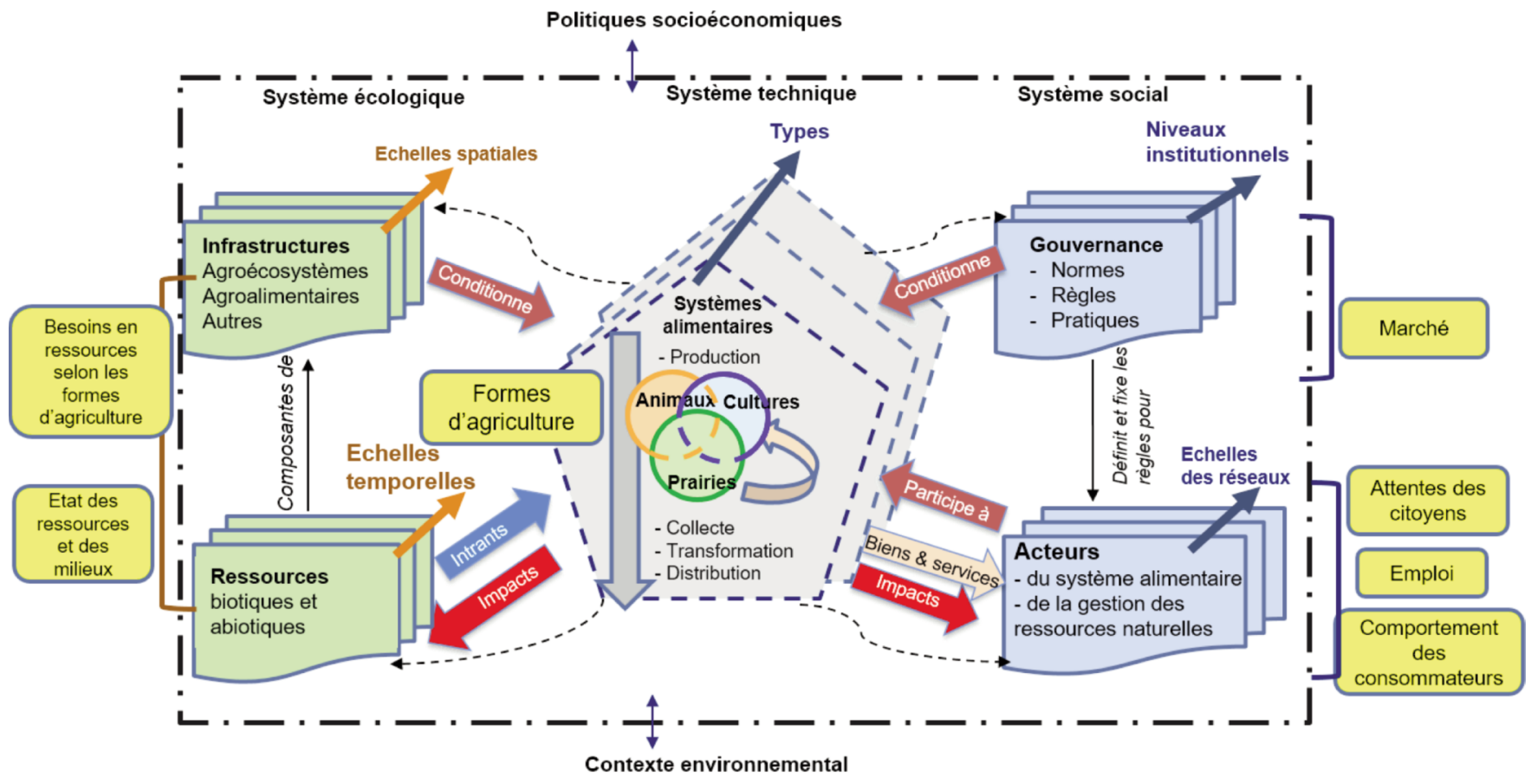

les systèmes écologiques et techniques se produisent à différentes échelles de temps au niveau du champ (par exemple, l'effet cumulatif des techniques de gestion du sol sur la fertilité du sol) et à des niveaux plus élevés (par exemple, cascade d'azote au niveau du paysage) (Duru et al 2015b).

Le système social comprend les acteurs du système alimentaire : les agriculteurs, les acteurs des filières jusqu'à la distribution, mais aussi les consommateurs et ceux impliqués dans la gestion des ressources naturelles (figure 1). Suivant les territoires et systèmes alimentaires, ces acteurs sont totalement ou partiellement inscrits dans le territoire analysé. Les institutions agissent sur le comportement des acteurs des systèmes techniques au travers de la définition de normes, de standards, de règlements/lois, mais aussi par la fixation de taxes et de subventions. Les consommateurs impactent les systèmes techniques au travers de la quantité et de la nature des produits animaux qu'ils achètent. Les citoyens, au travers de leur sensibilité à tel ou tel enjeux (bienêtre animal, sécurité alimentaire, pollutions) peuvent exercer un lobbying auprès des institutions en charge des réglementations.

Ces trois systèmes - écologique, technique (les élevages et leur inscription dans les filières) et social - interagissent entre eux, et ce à des échelles locales et globales. Le comportement des acteurs économiques est pour partie défini par des modalités de gouvernance élaborées à différentes échelles institutionnelles, région, États et Europe. Les interactions entre sous-systèmes se font entre niveaux et domaines au travers des processus biophysiques et socioéconomiques. Ainsi, l'état des ressources à l'échelle du paysage (eau, biodiversité) dépend de l'utilisation des terres. Il affecte les processus biophysiques à l'échelle du champ (eau disponible et régulations biologiques) et est sous l'influence des politiques environnementales aux échelles régionales et nationales (ex. politique de régulation de la qualité de l'eau). Les marchés locaux et les institutions, les filières (produits avec labels) ont différents degrés d'interdépendance (Therond et al 2017). Les interactions entre les domaines écologiques, économiques et sociaux déterminent les pratiques agricoles. Les encadrés jaunes de la figure 1 représentent quelquesuns des critères retenus pour l'analyse des impacts et services que nous proposons dans la section suivante.

Considérer l'élevage comme un système socio-écologique permet de transformer une liste de critères d'évaluation (tableau 1) en une représentation fonctionnelle facilitant l'analyse de la manière dont les effets positifs et négatifs de l'élevage et de la consommation de produits animaux sont reliés entre eux, et ce dans différents domaines. Il est aussi possible de montrer que les acteurs qui bénéficient des effets positifs peuvent ne pas être les mêmes que ceux qui subissent des effets négatifs. Les arbitrages sociaux pour traiter d'antagonismes entre services peuvent se faire au niveau local dans des dispositifs formalisés (par exemple pratiques d'élevage et pollution des eaux par les nitrates), ou bien à un niveau plus global et de manière plus « diffuse » et lente, par exemple au travers du changement de comportement des consommateurs.

\section{2 / La grange : une représenta- tion simplifiée de l'élevage dans les territoires}

En nous inspirant des conceptualisations précédemment présentées, nous proposons une représentation intégrative mais simplifiée des services et impacts rendus par l'élevage dans un territoire. Celle-ci se fonde sur une représentation des caractéristiques du système socioécologique (matrice paysagère à l'origine des services écosystémiques, valeur monétaire et emplois générés...), dans lequel les systèmes d'élevage interagissent avec cinq interfaces précédemment discutées : les marchés, le travail et l'emploi, les intrants, l'environnement et le climat, et les enjeux sociaux et culturels. Ces interfaces recouvrent les domaines d'évaluation annoncés dans le tableau 1 et la figure 1. Cette représentation est appelée « grange » (figure 2) en référence à la forme du pentagone central qui tout comme dans la figure 1, figure le territoire (ou le système) d'élevage analysé. Elle peut se décliner à différents niveaux d'organisation : un système d'élevage (exploitation ou coordination d'exploitations, Benoit et Méda 2017, ce numéro), un territoire (Dourmad et al 2017, Ryschawy et al 2017, ce numéro) ou une filière entière à l'échelle d'un pays (Delaby et al 2017, ce numéro). Elle 
permet de dresser un diagnostic de l'état actuel de l'élevage au sein d'un territoire, en caractérisant ses atouts et les points de vigilance à considérer dans les dynamiques d'innovation en émergence.

\section{a) Grille de lecture de la grange}

La grange commentée ci-dessous est celle des zones fromagères AOP du Massif central (figure 2). Le pentagone central représente le territoire d'élevage avec ses éléments paysagers (haies) et les activités agro-industrielles (industries agroalimentaires, méthaniseur) sur lesquelles s'appuie l'élevage. Celui-ci est décrit par ses caractéristiques : espèces et densité animales, modes d'alimentation (animal vert : au pâturage et à base de fourrages, ocre : rations à base d'aliments concentrés dont l'ensilage de maïs). Le mode d'usage des terres est représenté par un parcellaire à deux nuances de vert pour symboliser la diversité des prairies permanentes et la présence de prairies temporaires, et à deux nuances de jaune pour représenter la diversité des rotations culturales. Dans les espaces urbains et péri-urbains (Delfosse et al 2017, ce numéro), les surfaces artificialisées sont représentées en gris.

Sur chaque interface, des pictogrammes explicités sous chaque grange symbolisent les éléments concernés par ces interfaces. La bourse symbolise la création de valeur monétaire par vente des produits et est donc positionnée sur l'interface « marchés ». Selon les territoires, les systèmes bénéficient d'autres sources de revenus (MAE, ICHN, aides à l'agriculture biologique...) qui sont indiquées sous les livres qui représentent le cadre réglementaire (hors cahiers des charges animaux inclus dans le pictogramme « produits sous signes de qualités »). La poignée de main symbolise une coordination structurée entre les acteurs des filières ou du territoire. Certains pictogrammes sont de couleur variable selon que l'impact de l'élevage y est positif ou négatif : c'est par exemple le cas du pictogramme qui représente la qualité de l'eau, bleue lorsqu'elle n'est pas affectée par l'activité d'élevage, grise lorsqu'il y a un risque de pollution par les pesticides ou d'eutro-phisation.

La nature et l'ampleur des effets sont représentées par une flèche sortante plus ou moins large et dont la couleur indique que ceux-ci sont positifs (flèche verte), négatifs (rouge) ou mitigés (hachures); dans ce cas, l'effet dominant borde la flèche. Sur l'interface travail et emploi, nous distinguons les emplois directs en élevage des emplois indirects (IAA, tourisme, flèche vers personnage en blanc). Les flèches entrantes ont un sens spécifique à chaque interface. Sur l'interface intrants, la flèche rouge entrante

Figure 2. La " grange » : représentation graphique du cadre d'analyse des territoires ou des systèmes d'élevage.

Le pentagone central figure un territoire d'élevage (dans cet exemple les zones fromagères AOP du Massif central) et ses principales caractéristiques. Le mode d'usage des terres est représenté par un parcellaire à deux nuances de vert pour symboliser la diversité des prairies permanentes et la présence de prairies temporaires, et à deux nuances de jaune pour représenter la diversité des rotations culturales. Dans ce territoire, l'élevage interagit avec cinq interfaces, les côtés du pentagone, qui découlent du choix de mettre en exergue ses interactions avec l'ensemble des composantes du système socio-écologique. La signification des pictogrammes utilisés est rappelée sous chaque grange. La nature et l'ampleur des effets sont visualisées par une flèche sortante, plus ou moins large, dont la couleur indique s'ils sont positifs (vert), négatifs (rouge) ou mitigés (hachures). Des flèches entrantes peuvent symboliser les effets délocalisés de l'alimentation animale (intrants), la vulnérabilité aux fluctuations des prix (marchés), les services rendus par les écosystèmes (couleur verte, interface environnement-climat) ou la pression de facteurs environnementaux (couleur rouge).

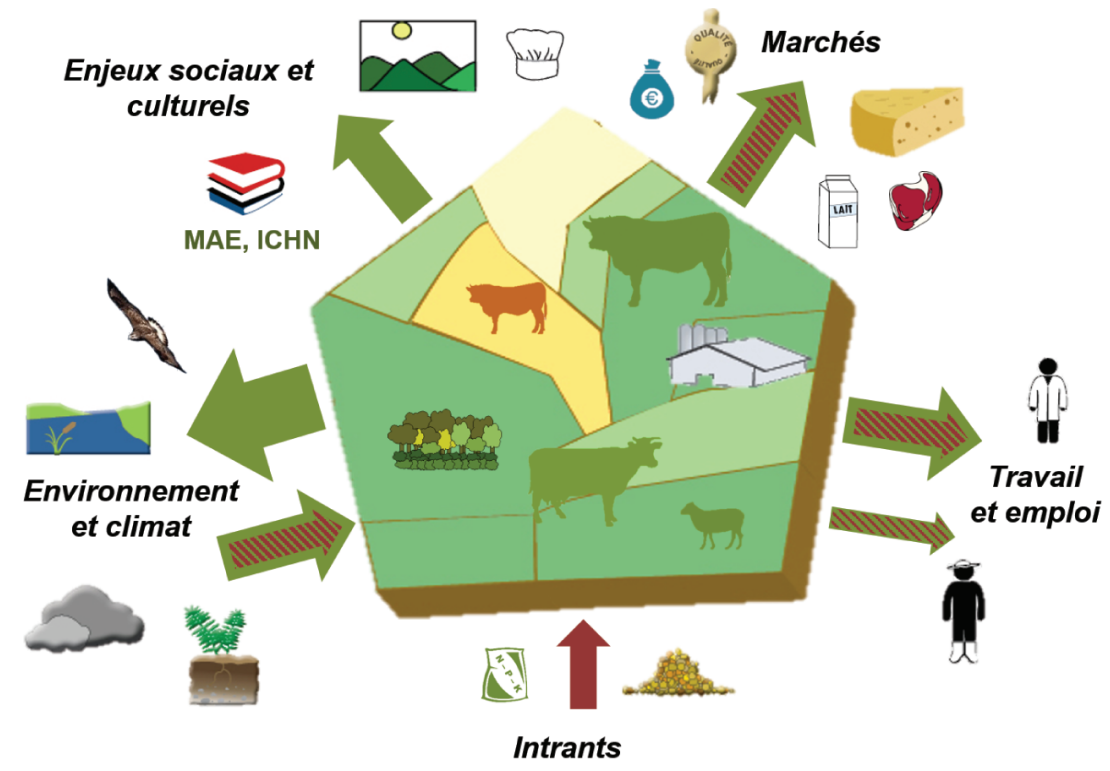

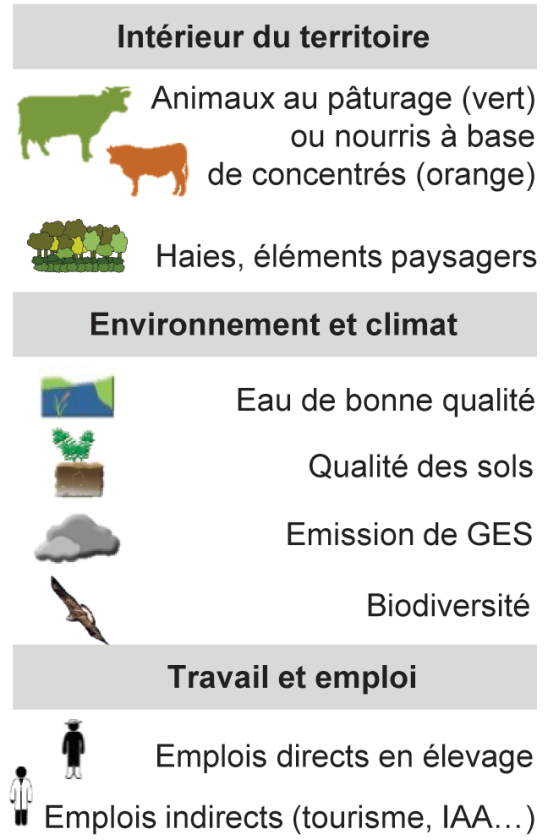

traduit l'importance que revêt l'utilisation d'intrants exogènes dans le système ; elle symbolise ainsi les effets délocalisés de l'élevage. Dans certains cas, cette flèche peut être hachurée de vert pour signifier que l'élevage importe et valo-

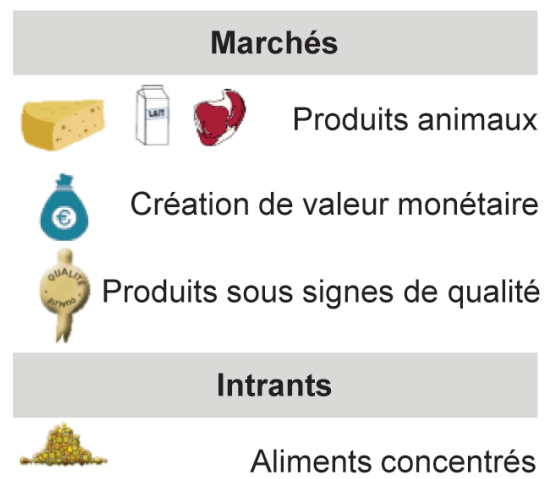

Engrais, pesticides, énergie

Enjeux sociaux et culturels

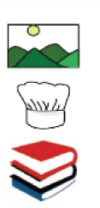

Esthétique paysagère

Patrimoine gastronomique

Aspects réglementaires et juridiques

rise des coproduits ou des déchets de l'alimentation humaine. Les flèches à l'intérieur du territoire symbolisent les interactions locales qui permettent de réduire les importations d'intrants, par exemple les échanges entre céréaliers et 
éleveurs (Ryschawy et al 2017), l'utilisation de cultures locales pour alimenter les animaux (Benoit et Méda 2017), ou la valorisation des déjections animales pour fertiliser cultures et prairies après méthanisation (Dourmad et al 2017). Sur l'interface marchés, la flèche entrante signifie une sensibilité de ce type d'élevage aux fluctuations du marché (coût des matières premières, tensions sur les prix des produits) lorsqu'elle est rouge, ou à l'inverse des opportunités (valorisation en circuits courts) lorsqu'elle est verte. Sur l'interface « environnement, climat », la flèche entrante signifie que les systèmes d'élevage bénéficient de services intrants (flèche verte) ou subissent la pression de facteurs environnementaux (risque de prédation, de contamination par l'avifaune sauvage, sécheresse...; flèche rouge). Chaque grange est accompagnée d'un tableau (tableau 2) qui synthétise les effets positifs et négatifs de l'élevage relatifs aux cinq interfaces. Notons enfin que les granges sont surtout à considérer relativement les unes par rapport aux autres ; l'absence d'un pictogramme ou d'une flèche ne signifie pas une absence d'effet, mais plutôt que celuici est négligeable comparé à d'autres territoires.

\section{b) Analyse des atouts et des limites de la grange}

Appliquée dans une gamme de territoires d'élevage (comme cela est réalisé dans les cas d'étude présentés dans les articles de ce numéro spécial), cette représentation simplifiée permet de visualiser les atouts et les pressions qui s'exercent sur ces territoires, et ainsi de les comparer. Elle constitue ainsi un outil pédagogique qui révèle qu'aucun territoire d'élevage n'est source que de services ou à l'inverse que d'impacts négatifs. À l'instar du travail de Ryschawy et al (2015) sur les services rendus par l'élevage, ce mode de représentation permet de visualiser que certains territoires sont très orientés vers la fourniture de services d'approvisionnement et de vitalité territoriale (Dourmad et al 2017), alors que d'autres produisent majoritairement des services de qualité environnementale et des services culturels (Vollet et al 2017, LemauvielLavenant et Sabatier 2017, ce numéro). La comparaison des granges permet également d'identifier des territoires dans lesquels l'élevage est susceptible de fournir des bouquets de services plus " équilibrés ». Elle met aussi en avant des grandes tendances dans les arbitrages entre ces différents impacts et services, que ce soit entre les grands types de territoires d'élevage ou au sein de chaque grand type. Ces arbitrages concernent principalement l'arbitrage entre la production d'une part et l'environnement et les dimensions socio-culturelles d'autre part.

Cette représentation synthétique, comme toute représentation visuelle, a une portée heuristique limitée, car l'ensemble des interactions ne peuvent pas être représentées. Malgré le potentiel de la grange pour représenter des niveaux d'organisation emboités, ceux-ci ne peuvent également être représentés dans un unique schéma. La largeur des flèches et la taille des pictogrammes est « à dire d'experts » à la différence des flux qui ont par exemple pu être quantifiés dans la cascade de l'azote (Billen et al 2015) ou dans des approches de métabolisme territorial (Bonaudo et al 2015). Ceci résulte du fait que certaines des variables représentées sur les côtés de la grange, telles que la perception de l'esthétique paysagère, sont difficilement quantifiables. Par ailleurs, des données quantitatives ne sont pas toujours disponibles à l'échelle des entités territoriales considérées ; c'est par exemple le cas des importations de concentrés ou d'éléments fertilisants ou des émissions de GES liées à l'élevage. Un atout majeur de la grange est donc de représenter dans un même schéma des variables qui ne s'exprimeraient pas dans les mêmes unités, et dont la plupart sont difficilement quantifiables au grain considéré. Par ailleurs, l'exercice de construction des granges ouvre un espace de dialogue entre les experts du territoire concerné et permet l'expression de points de vue divergents ; la représentation finale traduit ainsi le compromis qui a été obtenu entre les experts impliqués. Nous avons privilégié une focalisation sur l'emprise spatiale du système étudié plutôt que temporelle. Toutefois, notre mode de représentation ne rend pas toujours bien compte de la diversité des formes d'élevage qui y coexistent, et fait principalement ressortir le bouquet de services et les impacts négatifs générés par les systèmes dominants. Cette critique rejoint celle qui peut être formulée à l'encontre des cartographies de services

Tableau 2. Impacts positifs et négatifs associés aux territoires fromagers AOP du Massif central, relatifs aux cinq interfaces (exemple de tableau synthétique qui accompagne chaque grange).

\begin{tabular}{|c|c|c|}
\hline $\begin{array}{l}\text { Interfaces } \\
\text { étudiées }\end{array}$ & Effets positifs & Effets négatifs \\
\hline Intrants & - Autonomie fourragère élevée & $\begin{array}{l}\text { - Effets délocalisés de la production d'aliments } \\
\text { concentrés (par exemple déforestation au Brésil } \\
\text { et en Argentine (Boerema et al 2016) } \\
\text { - Fertilisation minérale des prairies }\end{array}$ \\
\hline $\begin{array}{l}\text { Travail } \\
\text { et Emploi }\end{array}$ & $\begin{array}{l}\text { - Emplois directs en élevage assez nombreux } \\
\text { (petites structures) } \\
\text { - Maintien du tissu rural } \\
\text { - Emplois indirects (transformation, tourisme) }\end{array}$ & $\begin{array}{l}\text { - Organisation variable des filières } \\
\text { - Assez faible rémunération du travail agricole }\end{array}$ \\
\hline Marchés & $\begin{array}{l}\text { - Produits avec un potentiel de valeur ajoutée } \\
\text { (SIQO, circuits courts) }\end{array}$ & $\begin{array}{l}\text { - Saisonnalité de la production à l'herbe } \\
\text { - Valorisation limitée dans le territoire pour les } \\
\text { broutards }\end{array}$ \\
\hline $\begin{array}{l}\text { Enjeux sociaux } \\
\text { et culturels }\end{array}$ & $\begin{array}{l}\text { - Bonne image des systèmes et des produits } \\
\text { - Qualité nutritionnelle des produits à l'herbe } \\
\text { - Esthétique des paysages. Patrimoine } \\
\text { gastronomique }\end{array}$ & \\
\hline $\begin{array}{l}\text { Environnement } \\
\text { et climat }\end{array}$ & $\begin{array}{l}\text { - Biodiversité (prairies permanentes, } \\
\text { infrastructures paysagères, habitats remarquables) } \\
\text { - Séquestration C par prairies } \\
\text { - Préservation qualité des eaux }\end{array}$ & $\begin{array}{l}\text { - Émissions élevées } \mathrm{CH}_{4} \text { par } \mathrm{kg} \text { de produit } \\
\text { - Sensibilité aux aléas climatiques et aux ravageurs } \\
\text { (rats taupiers) }\end{array}$ \\
\hline
\end{tabular}


(Dumont et al 2017b, ce numéro). La question des temporalités et de la coexistence de différents systèmes au sein d'un territoire sont cependant abordées de manière qualitative lorsque sont examinées différentes voies de progrès pour l'élevage. Nous discutons dans la section suivante les clefs utilisées à ces fins.

\section{3 / Caractériser la diversité des élevages pour les impacts et services}

\section{1 / Différents paradigmes pour réduire les impacts et augmenter les services}

La grange fournit une représentation synthétique des impacts et services de l'élevage et des filières. Elle permet de représenter plusieurs façons de produire et de s'ancrer dans un territoire, appelées formes d'élevage. Therond et al (2017) proposent une grille d'analyse structurée autour de deux grandes dimensions pour clarifier le positionnement relatif des formes d'élevage : la nature et l'origine des intrants mobilisés dans les systèmes de production et la nature des contextes socioéconomiques dans lesquels ils sont insérés.

La première dimension est basée sur le poids relatifs des deux grands types $\mathrm{d}$ 'intrants : i) exogènes à l'élevage, qu'ils soient chimiques (ex. engrais de synthèse, pesticides, médicaments) ou biologiques (ex. stimulateur de la santé des plantes et des animaux, matière organique) et ii) les services écosystémiques intrants (endogènes) dont le niveau détermine celui de l'autonomie en intrants des systèmes d'élevage (encadré 1). Les principes pour faire face aux enjeux de durabilité environnementale diffèrent : dans les systèmes basés sur les intrants chimiques exogènes il s'agit de maitriser les pollutions, les déchets et la rareté des ressources ; pour ceux basés sur les intrants biologiques, il s'agit de réduire l'écotoxicité des intrants et ainsi répondre aux enjeux de santé de l'écosystème et de l'Homme; enfin, dans les systèmes basés sur les services écosystémiques la priorité est mise sur la valorisation des ressources naturelles pour obtenir une plus grande autonomie vis à vis des intrants et de l'alimentation des animaux.

La seconde dimension permet de distinguer les contextes socio-économiques d'achat d'intrants, de commercialisation des produits agricoles voire de gestion des ressources naturelles qui sous-tendent le fonctionnement biotechnique des systèmes d'élevage (composantes intrants et marché du pentagone de la figure 2). Deux grands types de contextes socio-économiques peuvent être distin- gués. Le premier type correspond aux systèmes alimentaires industrialisés structurés par des marchés mondialisés de produits génériques et standardisés, de composés issus du fractionnement des matières premières ou d'intrants. Le second type englobe les contextes socio-économiques qui favorisent un ancrage territorial des systèmes de production. Nous distinguons ici trois sous-types de contextes favorisant cet ancrage $: i$ ) les économies circulaires qui visent à boucler localement le cycle des matières et de l'énergie (par ex méthanisation), ii) les systèmes alimentaires alternatifs et, iii) les projets de développement territorial intégré. Ces trois types de dynamiques sont nés en réaction aux effets négatifs des systèmes alimentaires industrialisés sur l'environnement, la qualité des produits ou, la santé humaine, ou encore pour répondre à des enjeux locaux (ex. souveraineté, relocalisation). Une manière de caractériser ces différents contextes socio-économiques est d'analyser dans quelle mesure les relations sociales sont organisées pour réguler les comportements purement économiques des acteurs (composantes enjeux sociaux et culturels du pentagone de la figure 2). On parle alors de degré d'intégration territoriale (territorial embeddedness). Penker (2006) identifie ainsi trois dimensions clés de l'intégration territoriale des activités économiques fonction de la prise en compte $: i$ ) des relations sociales entre les agents du système alimentaire (ex. confiance, transparence, équitabilité) ; ii) de l'origine géographique des produits ou des modes de production; iii) des caractéristiques environnementales associées aux produits, c'est-à-dire la prise en compte des impacts sur l'environnement et la santé des pratiques agricoles ou des systèmes alimentaires. Ces distinctions permettent de caractériser la diversité des contextes socio-économiques des systèmes de production agricole (Therond et al 2017).

Ce cadre d'analyse à deux dimensions (figure 3) permet de dépasser les limites des classifications basées sur des dénominations générales telles que : agriculture biologique, conventionnelle, de précision, intégrée, climato-intelligente, etc. En effet, la plupart de ces catégories englobent une grande diversité de modes de production plus ou moins basés sur les intrants exogènes et les services écosystémiques et de contextes socioéconomiques. De ce fait, elles ne permettent pas de distinguer les systèmes en fonction de leurs performances environnementales et socio-économiques.

\section{2 / Formes d'élevage : diversité des cas d'étude retenus et des voies de progrès examinées dans l'Esco}

En analysant les systèmes d'élevage suivant le poids relatif des intrants d'une part et leur contexte socio-économique (mondialisés vs territorialisés) d'autre part, six archétypes de formes d'élevage peuvent être définis (figure 3), (Therond et al 2017) :

- Une forme d'élevage dans laquelle les systèmes de production sont basés sur des intrants chimiques (engrais de synthèse, produits phytosanitaires et médicaments), généralement non autonome en protéines, voire systèmes d'élevage tout ou partie hors sol, et inscrits dans des systèmes alimentaires mondialisés ;

- Deux formes d'élevage basées sur les intrants biologiques, ayant un degré d'autonomie alimentaire généralement plus élevé, et insérées dans des systèmes alimentaires mondialisés ou dans des économies circulaires, le plus souvent pour les intrants, y compris les aliments pour le troupeau ;

- Trois formes d'élevage basées sur l'utilisation des services écosystémiques intrants intégrées dans : $i$ ) des systèmes alimentaires mondialisés lorsque le contexte territorial ne se prête pas à valoriser un ancrage territorial des façons de produire ; ii) des économies circulaires combinées à des systèmes alimentaires alternatifs ; iii) un projet territorial intégré basé sur les complémentarités entre économie circulaire, systèmes alimentaires alternatifs et gestion collective de paysage multiservices.

Dans une première forme d'élevage (1), les systèmes d'élevage spécialisés, produisant majoritairement à partir d'intrants chimiques et faiblement autonomes en protéines, sont fortement insérés dans des systèmes alimentaires d'échanges de matières premières structurés par les prix des cours mondiaux (quadrant inférieur gauche de la figure 2). Étant donné qu'ils sont intégrés dans des systèmes alimentaires fonctionnant à grande échelle, ces systèmes d'élevage sont souvent peu connectés aux enjeux et stratégies locales de gestion des ressources naturelles, ce qui entraîne souvent des conflits pour la gestion de ressources (eau pour l'irrigation, qualité de l'eau). Cette forme d'élevage fait partie de la forme d'agriculture dominante en Europe occidentale (Levidow et al 2014). Les cas d'étude de la Bretagne et de la Catalogne illustreront ce modèle (Dourmad et al 2017). Mais les systèmes de polyculture élevage reposant sur peu d'intégration spatiotemporelle entre les ateliers s'inscrivent dans ce modèle (Ryschawy et al 2017).

Les deux formes d'élevage basées sur l'utilisation d'intrants biologiques interagissent également avec les chaînes d'approvisionnement alimentaire mondialisées pour la fourniture d'intrants biologiques (par exemple bio-stimulants et 
Figure 3. Six formes d'agriculture selon la nature et l'origine des intrants utilisés dans les élevages (axe Y) et selon leur insertion dans des systèmes alimentaires mondialisés et dans les dynamiques territoriales (axe X), (adapté de Therond et al 2017).

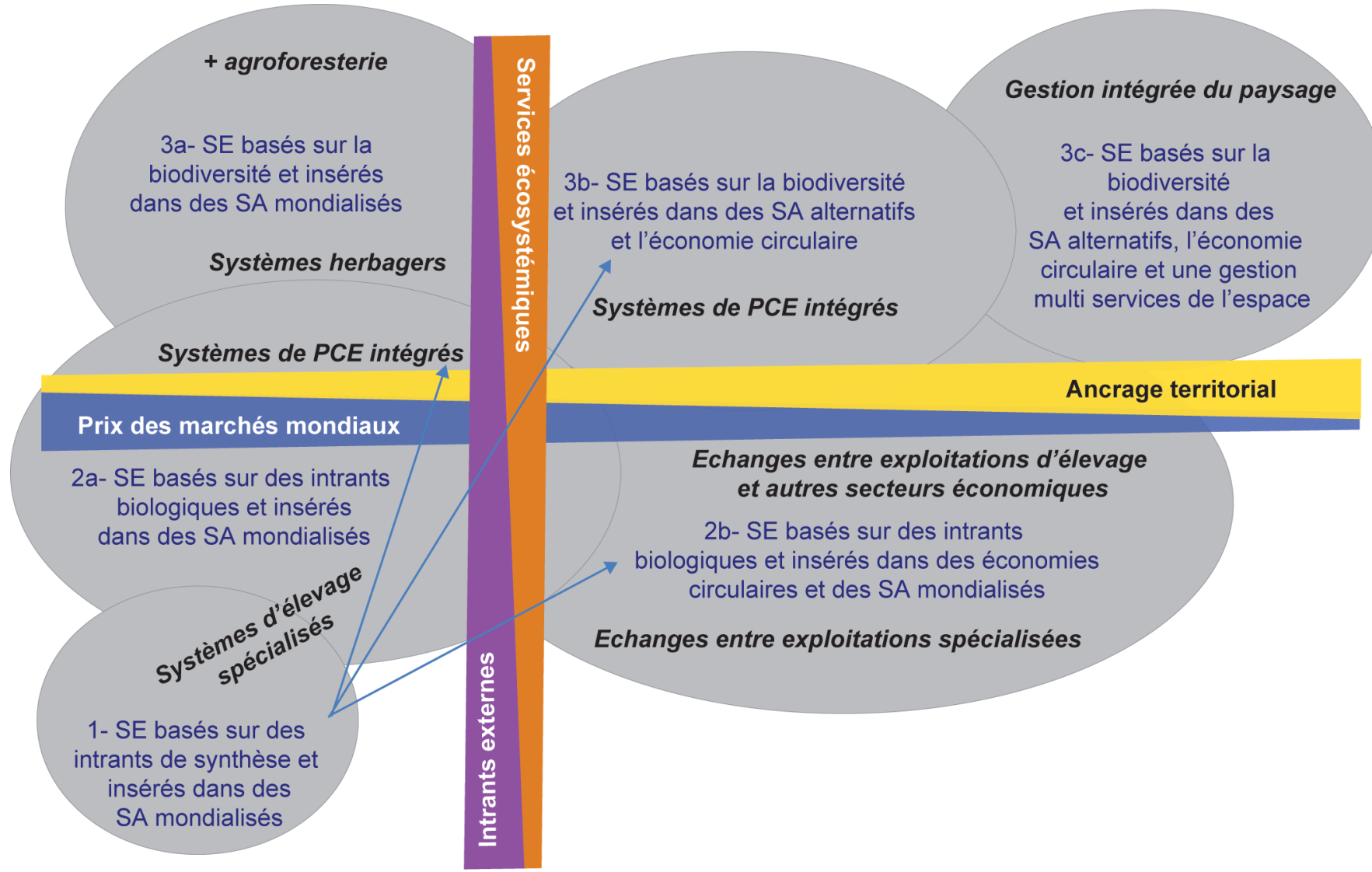

SE : Système d’Élevage ; SA : Système Alimentaire ; PCE : PolyCulture-Élevage

Les flèches indiquent des trajectoires possibles d'évolution ou de transformation des systèmes de production d'une forme à une autre avec ici l'exemple des systèmes de polyculture élevage dont les ateliers sont juxtaposés (quadrant en bas à gauche), peuvent réduire les intrants en procédant à des échanges entre exploitations spécialisées (quadrant en bas à droite) ou en intégrant mieux les deux ateliers au sein d'une exploitation ou encore en s'inscrivant dans des dynamiques territoriales (quadrant en haut à droite)

bio-pesticides) et la commercialisation des produits (2a) (figure 3, quadrant inférieur gauche). Toutefois, dans la deuxième forme $(2 \mathrm{~b})$ le fonctionnement des élevages peut aussi fortement reposer sur une économie circulaire (par exemple, échanges de matières, fourrages, pailles, fumiers, entre exploitation spécialisées en élevage et en grande culture), ce qui permet de remplacer tout ou partie des intrants chimiques par des intrants biologiques et d'offrir des opportunités de diversification (par exemple, la production de biomasse pour la bioénergie, comme dans le cas d'étude Allemand (Dourmad et al 2017), (figure 3, quadrant inférieur droit). L'émergence d'échanges entre exploitations spécialisées en grande culture et en élevage s'inscrit aussi dans ce modèle (Ryschawy et al 2017). Le développement d'économies circulaires est encouragé par les politiques européennes et les acteurs de l'agroindustrie (Levidow et al 2014).

Les trois formes d'élevage basées sur la gestion de la biodiversité se distinguent selon les contextes socio-économiques dans lesquels elles s'inscrivent. Dans ces formes, la diversité des ressources fourragères (prairies, parcours), associées à une diversité de cultures permet de fournir des services écosystémiques intrants (encadré 2). Le plus fort lien au sol, éventuellement combiné à une diversité d'espèces animales, contribue à une autonomie alimentaire des troupeaux plus élevée que celles atteignable dans les formes 1 ou $2 \mathrm{a}$. La première de ces formes basées sur la biodiversité (3a) correspond à des systèmes d'élevage herbagers relativement autonomes pour l'alimentation dans des zones à forte concentration d'animaux mais avec alignement de la production laitière sur la pousse de l'herbe et des niveaux de production modestes comme en Irlande (Delaby et al 2017), voire à de l'élevage combiné à de l'agriculture de conservation ou de l'agroforesterie (figure 3 , quadrant supérieur gauche). Les systèmes d'élevage herbagers à moyenne densité animale dont les produits sont vendus au cours mondial s'inscrivent aussi dans ce modèle. Par contre, lorsque les produits de l'élevage s'insèrent dans des systèmes alimentaires alternatifs et des économies circulaires au niveau local ou régional via des filières adaptées (Vollet et al 2017), ces systèmes d'élevage herbagers s'inscrivent dans la forme $3 \mathrm{~b}$, correspondant souvent à l'utilisation de ressources diversifiées (figure 3, quadrant supérieur droit). C'est également le cas d'élevages ovins bio et de granivores avec fort lien au sol dont les produits sont valorisés avec des labels (Benoit et Méda 2017). La forme 3c intègre en plus la gestion collective de modes d'utilisation des sols agricoles et non agricoles pour le développement d'un bouquet de services à l'échelle du territoire (quadrant supérieur droit de la figure 3). Les territoires à forts enjeux naturels où l'élevage rend des services de régulation et de préservation de la biodiversité et des paysages (LemauvielLavenant et Sabatier 2017), de même que les élevages situés dans les espaces périurbains (Delfosse et al 2017), s'inscrivent le plus souvent dans les formes $3 \mathrm{~b}$, voire $3 \mathrm{c}$ lorsque la dimension collective pour l'utilisation de l'espace et/ou la commercialisation des produits est 
forte. Le premier modèle basé sur la biodiversité (3a) correspond souvent à des niches dans les pays européens ; mais des exceptions sont observées comme l'élevage laitier en Irlande. Le poids des deux autres formes ( $3 b$ et $3 c$ ) est actuellement encore plus limité en termes de volumes de produits.

La classification présentée permet de distinguer les élevages sur des bases plus génériques et plus précises que les classifications usuelles : conventionnels vs bio; filières longues vs circuits courts, élevages avec ou sans labels. Ainsi, les exploitations de polyculture élevage peuvent correspondre à la plupart des formes, tout comme les élevages produisant avec labels, selon respectivement l'intensité des interactions entre ateliers et des services écosystémiques associés, ainsi que du degré d'ancrage dans le territoire (Moraine et al 2016). L'analyse des services et des impacts passe donc par l'explicitation des modes de production (degré d'autonomie, part des services écosystémiques intrants) et du degré d'ancrage des élevages dans les territoires plus que par le rattachement à telle ou telle catégorie de signe officiel de qualité, cahier des charges ou technologies utilisées. Cette représentation permet tout autant de décrire les formes d'élevage courantes que celles émergentes. Dans la plupart des territoires, différents formes d'agriculture et d'élevage coexistent. Mais lorsque l'élevage est caractérisé à partir de bases de données portant sur de grands territoires (département ou région), seuls les systèmes dominants sont identifiables (Dumont et al 2017b). Par contre, au sein de ces mêmes territoires, les études de cas permettent de caractériser des élevages innovants dans leur mode de production ou dans leur relation avec le territoire, et ce, seul, en petits groupes ou dans des collectifs plus larges. Ces deux niveaux d'analyse seront présentés dans la plupart des cas d'étude.

Cette représentation permet également de caractériser les types de transition (Caron et al 2014) entre ces formes selon qu'elles n'engendrent que des évolutions incrémentales (substitution des intrants), ou, nécessitent des transformations plus profondes (figure 3 , flèches courbes indiquant des trajectoires). Par exemple, les élevages situés dans les territoires sous tension environnementale (Dourmad et al 2017) peuvent accroître leur durabilité par mobilisation des leviers de l'économie circulaire, par développement de la part de l'herbe et la recherche d'autonomie, ou bien encore par des changements plus radicaux basés sur les modes de commercialisation alternatifs, éventuellement sur la base de labels (bio...). Les élevages situés dans les territoires herbagers (Vollet et al 2017) peuvent renforcer leur autonomie en intrants par accroissement de la biodiversité (légumineuses, agroforesterie), mais ils peuvent aussi s'organiser pour produire sous signes de qualité afin de valoriser l'alimentation à l'herbe dans le cas des ruminants ou faire reconnaître un plus grand lien au sol pour les monogastriques. Les élevages situés dans les territoires de polyculture élevage (Ryschawy et al 2017) peuvent renforcer l'intégration entre les deux ateliers au sein de l'exploitation, ou bien via le développement d'échanges entre exploitations spécialisées au sein d'un territoire, soit dans le cadre d'économie circulaire, soit en accroissant la biodiversité cultivée ou bien encore au travers d'engagement dans des cahiers des charges mentionnant un plus fort ancrage territorial. Les productions animales sous signe de qualité (Benoit et Méda 2017) peuvent quant à elles valoriser et combiner des ancrages liés aux spécificités du milieu naturel, aux façons de produire par le lien au sol ou l'organisation sociale. Dans tous les cas, les critères d'évaluation considérés seront ceux identifiés dans la grange ( $\$ 3.2$.).

\section{Conclusion}

Nous avons proposé une représentation graphique simplifiée de l'élevage et de son ancrage territorial sur la base d'une analyse de la littérature scientifique relative aux systèmes socio-écologiques, notamment la plus récente qui inclut les systèmes anthropisés et les activités économiques. La " grange » permet d'abord de visualiser les caractéristiques de l'élevage et les interactions associées dans un territoire et avec son environnement suivant cinq grands types d'interfaces qui recouvrent les différents piliers de la durabilité et permet de prendre en compte les effets locaux et les effets délocalisés de l'élevage. Du point de vue biophysique, elle met l'accent sur les interactions entre cultures (fourragères ou non, de vente ou autoconsommées), prairies et animaux (ruminants ou monogastriques) de l'échelle de l'exploitation à celle du territoire et sur le degré de circularité des matières et le niveau potentiel de fourniture de services écosystémiques, notamment par les prairies. Du point de vue socio-économique elle permet de prendre en compte explicitement la nature et les volumes de produits échangés sur les marchés, le travail et l'emploi, ainsi que les dimensions sociétales et culturelles liées à l'élevage. Sur la base de l'analyse des relations entre les caractéristiques de ses cinq interfaces, la grange permet une analyse fonctionnelle de l'élevage dans un territoire afin d'en déterminer les atouts et les points de vigilance. Pour typer la diversité des formes d'élevages dans un territoire, nous avons retenu une classification basée sur la distinction (i) du poids relatif des intrants exogènes, chimiques ou biologiques, et des services écosystémiques dans le fonctionnement des systèmes d'élevage, et (ii) le niveau d'insertion de ces derniers dans les systèmes alimentaires mondialisés vs les dynamiques territorialisées. Cette grille d'analyse permet de positionner les différents cas d'études présentés dans les articles suivants de ce numéro spécial. Plus largement, elle permet d'aider à caractériser les types de transition de ces formes d'élevage afin d'augmenter la gamme de services qu'elles fournissent et de réduire leurs impacts négatifs sur l'environnement.

\section{Références}

ANSES, 2016. AVIS et RAPPORTS de l'Anses relatifs à l'Actualisation des repères du PNNS : Révision des repères de consommations alimentaires. 192p.

Benoit M., Méda B., 2017. Enjeux et atouts des productions animales sous signe officiel de qualité pour répondre aux attentes sociétales. In : Numéro spécial, L'élevage en Europe : une diversité de services et d'impacts. Dumont B. (Éd). INRA Prod. Anim., 30, 381-394.

Billen G., Lassaletta L., Garnier J., 2015. A vast range of opportunities for feeding the world in 2050: trade-off between diet, $\mathrm{N}$ contamination and international trade. Environ. Res. Letters, $10,1-14$.

Boerema A., Peeters A., Swolfs S., Vandevenne F., Jacobs S., Staes J., Meire P., 2016. Soybean trade: Balancing environmental and socio-economic impacts of an intercontinental market. PLoS ONE, 11, 1-13.

Bommarco R., Kleijn D., Otts S.G., 2012. Ecological intensification: harnessing ecosystem services for food security. Trends in Ecology \&
Evolution, 1-9. http://doi.org/10.1016/j.tree.2012. 10.012

Bonaudo T., Billen G., Garnier J., Barataud F., Bognon S., Marty P., Dupre D., 2015. Le système agro-alimentaire : un découplage progressif de la production et de la consommation. In : Essai d'écologie territoriale. L'exemple d'Aussois en Savoie. Buclet N. (Éd). 157-178. CNRS Alpha. Paris, France. CNRS Éditions.

Caron P., Biénabe E., Hainzelin E., 2014. Making transition towards ecological intensifi- 
cation of agriculture a reality: the gaps in and the role of scientific knowledge. Curr. Opin. Environ. Sustainability, 8, 44-52.

Chardigny J., Walrand S., 2016. A How might oil seeds help meet the protein challenge ?Oleagineux Corps Gras Lipides.

Chaudhary A., Kastner T., 2016. Land use biodiversity impacts embodied in international food trade. Global Environ. Change, 38, 195-204.

Daily G., 1997. Nature's services: societal dependence on natural ecosystems. Edition Island Press, 412p.

d'Alexis S., Sauvant D., Boval M., 2014 Mixed grazing systems of sheep and cattle to improve live weight gain: a quantitative review. J. Agricult. Sci., 152, 655-666.

de Groot R.S., Alkemade R., Braat L., Hein L., Willemen L., 2010. Challenges in integrating the concept of ecosystem services and values in landscape planning, management and decision making. Ecol. Complexity, 7, 260-272.

Delaby L., Chatellier V., Dumont B., Horan B., 2017. L'Irlande, un territoire porté par l'élevage laitier dans des conditions de milieu favorable et de marchés incertains. In : Numéro spécial, L'élevage en Europe : une diversité de services et d'impacts. Dumont B. (Éd). INRA Prod. Anim., 30, 321-332.

Delfosse C., Dumont B., Hostiou N., 2017. Des services contrastés rendus par l'élevage dans les espaces urbains et périurbains européens. In : Numéro spécial, L'élevage en Europe : une diversité de services et d'impacts. Dumont B. (Éd). INRA Prod. Anim., 30, 395-406.

Dourmad J.Y., Delaby L., Boixadera J., Ortis C., Méda B., Gaigné C., Dumont B., 2017. Diversité des services rendus par les territoires à forte densité d'élevages, trois cas d'étude en Europe. In : Numéro spécial, L'élevage en Europe : une diversité de services et d'impacts. Dumont B. (Éd). INRA Prod. Anim., 30, 303-320.

Dumont B., Dupraz P., Sabatier R., Hercule J., Donnars C. 2017a. Pour toute recherche dans la base de données Une expertise scientifique collective analyse les rôles, impacts et services issus des élevages en Europe, Fourrages, 229, 63-76.

Dumont B., Ryschawy J., Duru M., Benoit M., Delaby L., Dourmad J.Y., Méda B., Vollet D., Sabatier R., 2017b. Les bouquets de services, un concept clé pour raisonner l'avenir des territoires d'élevage. In : Numéro spécial, Dumont B. (Éd). L'élevage en Europe : une diversité de services et d'impacts. INRA Prod. Anim., 30, 407-422.

Duru M., Therond O., 2015. Livestock system sustainability and resilience in intensive production zones: which form of ecological modernization? Regional Environmental Change, 15, 1651-1665.

Duru M., Therond O., Martin G., MartinClouaire R., Magne M., Justes E., Sarthou J.P., 2015a. How to implement biodiversity-based agriculture to enhance ecosystem services. Agron. Sustain. Dev., 35, 1259. http://doi.org/ $\underline{10.1007 / \mathrm{s} 13593-015-0306-1}$

Duru M., Moraine M., Therond O., 2015b. An analytical framework for structuring analysis and design of sustainable ruminant livestock systems. Anim., Frontiers, 5, 6-13.
FAO, Steinfeld H., Gerber P.J., Wassenaar T., Castel V., Rosales M., de Haan C., 2006. Livestock long shadow. Environmental issues and options. FAO, Rome, Italy, 390p.

Feng C., Ding S., Zhang T., Li Z., Wang D., Wang L., Peng F., 2016. High plant diversity stimulates foraging motivation in grazing herbivores. Basic Appl. Ecol., 17, 43-51. http://doi. org/10.1016/j.baae.2015.09.004

Foley J.A., Ramankutty N., Brauman K.A., Cassidy E.S., Gerber J.S., Johnston M., Mueller N.D., O'Connell C., Ray D.K., West P.C., Balzer C., Bennett E.M., Carpenter S.R., Hill J., Monfreda C., Polasky S., Rockstrom J., Sheehan J., Siebert S., Tilman D., Zaks D.P.M., 2011. Solutions for a cultivated planet. Nature, 478, 337-342.

Folke C., Carpenter S.R., Walker B., Scheffer M., Chapin T., Rockström J., 2010. Resilience thinking: integrating resilience, adaptability and transformability. Ecol. Society, 15.

Foran T., Butler J.R., Williams L.J. Wanjura W.J., Hall A., Carter L., Carberry P.S., 2014. Taking Complexity in Food Systems Seriously: An Interdisciplinary Analysis. World Dev., 61, 85-101.

Gerber P.J., Steinfeld H., Henderson B., Mottet A., Opio C., Dijkman J., Falcucci A., Tempio G., 2013. Tackling climate change through livestock - A global assessment of emissions and mitigation opportunities. FAO, Rome, Italy, $116 \mathrm{p}$

Gómez-Baggethun E., De Groot R., 2010 Natural capital and ecosystem services: the ecological foundation of human society. Ecosys. services, 105-121.

Haines-Young R., Potschin M., Kienast F. 2012. Indicators of ecosystem service potential at European scales: Mapping marginal changes and trade-offs. Ecol. Indicators, 21, 39-53.

Janzen H.H., 2011. What place for livestock on a re-greening earth? Anim. Feed Sci. Technol., 783-796.

Kremen C., Iles A., Bacon C., 2012. Diversified farming systems: an agroecological, systemsbased alternative to modern industrial agriculture. Ecol. Soc., 17, 44.

Lemauviel-Lavenant S., Sabatier R., 2017. Quand l'élevage est garant de la conservation de milieux patrimoniaux. In : Numéro spécial, L'élevage en Europe : une diversité de services et d'impacts. Dumont B. (Éd). INRA Prod. Anim., 30, 351-362.

Le Roux X., Barbault R., Baudry J., Burel F., Doussan I., Garnier E., Herzog F., Lavorel S., Lifran R., Roger-Estrade J., Sarthou J.P., Trommetter M., 2008. Agriculture et Biodiversité. Valoriser les synergies. Final report (Anglais).

Lescourret F., Magda D., Richard G., AdamBlondon A.F., Bardy M., Baudry J., Doussan I., Dumont B., Lefevre F., Litrico I., MartinClouaire R., Montuelle B., Pellerin S., Plantegenest M., Tancoigne E., Thomas A., Guyomard H., Soussana J.F., 2015. A socialecological approach to managing multiple agro-ecosystem services. Current Opinion Environ. Sustainability, 14, 68-75.

Levidow L., Pimbert M., Vanloqueren G., 2014 Agroecological research: conforming-or transforming the dominant Agro-Food Regime? Agroecol. Sustainable Food Syst., 38, 1127-1155.
Lindemann-Matthies P., Junge X., Matthies D., 2010. The influence of plant diversity on people's perception and aesthetic appreciation of grassland vegetation. Biological Conservation, 143, 195-202. http://doi.org/10.1016/j.biocon. 2009.10.003

Marshall G.R., 2015. A social-ecological systems framework for food systems research: accommodating transformation systems and their products. Int. J. Commons, 9, 881-908.

McGinnis M.D., Ostrom E., 2014. Social-ecological system framework: initial changes and continuing challenges. Ecol. Society, 19.

MEA - Millennium Ecosystem Assessment, 2005. Ecosystems and Human Well-Being: Multiscale Assessments: Findings of the SubGlobal Assessments Working Group. Island Press, 416p.

Moraine M., Duru M., Nicholas P., Leterme P., Therond O., 2014. Farming system design for innovative crop-livestock integration in Europe. Animal, 8, 1204-1217.

Moraine M., Duru M., Therond O., 2016. A social-ecological framework for analyzing and designing integrated crop-livestock systems from farm to territory levels. Renewable Agricult. Food Syst., FirstView, 1-14.

Ollion E., Ingrand S., Delaby L., Trommenschlager J.M., Colette-Leurent S., Blanc F., 2016. Assessing the diversity of trade-offs between life functions in early lactation dairy cows. Livest. Sci., 183, 98-107.

Penker M., 2006. Mapping and measuring the ecological embeddedness of food supply chains. Geoforum.37, 368-379. doi: 10.1016/j.geoforum.2005.09.001

Peyraud J.L., 2017. L'élevage contribue à la production durable de protéines. In: Le Demeter, Club Demeter, Paris, France, 363-388.

Pighin D., Pazos A., Chamorro V., Paschetta F., Cunzolo S., Godoy F., Messina V., Pordomingo A., Grigioni G., 2016. A Contribution of beef to human health: A Review of the role of the animal production systems. Scientif. World J., Article ID 8681491, 10p.

Pimentel D., 2003. Sustainability of meatbased and plant-based diets and the environment. Am. J. Clinical Nutr., 78, 660S-663S.

Rockstrom J., Steffen W., Noone K., Persson A., Chapin F.S., Lambin E., Lenton T.M., Scheffer M., Folke C., Schellnhuber H.J., Nykvist B., de Wit C.A., Hughes T., van der Leeuw S., Rodhe H., Sorlin S., Snyder P.K., Costanza R., Svedin U., Falkenmark M., Karlberg L., Corell R.W., Fabry V.J., Hansen J., Walker B., Liverman D., Richardson K., Crutzen P., Foley J., 2009. Planetary Boundaries: Exploring the Safe Operating Space for Humanity. Ecol. Society, 14, 32.

Ryschawy J., Tichit M., Bertrand S., Allaire G., Plantureux S., Aznar O., Perrot C., Guinot C., Josien E., Lasseur J., Aubert C., Tchakerian E., Disenhaus C., 2015. Comment évaluer les services rendus par l'élevage ? Une approche méthodologique sur le cas de la France. INRA Prod. Anim., 28, 23-37.

Ryschawy J., Benoit M., Hostiou N., Delfosse C., 2017. Quelles concurrences et synergies entre cultures et élevage dans les territoires de polyculture-élevage? In : Numéro spécial, 
L'élevage en Europe : une diversité de services et d'impacts. Dumont B. (Éd). INRA Prod. Anim., 30, 363-380.

Sabate J., Harwatt H., Soret S., 2016. Environmental Nutrition: A New Frontier for Public Health. Am. J. Public Health, 106, 815-821.

Therond O., Duru M., Roger-Estrade J., Richard G., 2017. A new analytical framework of farming system diversity to identify knowledge gaps in agronomy research. Agron. Sustain. Dev., 37, 21. https://doi.org/10.1007/s13593017-0429-7

Tilman D., Clark M., 2014. Global diets link environmental sustainability and human health. Nature, 515, 518-522.

Vallejo-Rojas V., Ravera F., Rivera-Ferre M.G., 2015. Developing an integrated framework to assess agri-food systems and its application in the Ecuadorian Andes. Regional Env. Change, First online, 1-15.

van der Linden A., Oosting S. J., van de Ven G.W.J., de Boer I. J. M., van Ittersum, M. K., 2015. A framework for quantitative analysis of livestock systems using theoretical concepts of production ecology. Agricult. Systems, 139, 100109. http://doi.org/10.1016/j.agsy.2015.06.007

van Zanten H.H.E., Meerburg B.G., Bikker P., Herrero M., de Boer I.J.M., 2016. Opinion paper: The role of livestock in a sustainable diet: a land-use perspective. Animal, 10, 547549

Vollet D., Huguenin-Elie O., Martin B., Dumont B., 2017. La diversité des services rendus par les territoires d'élevage herbagers fournissant des produits de qualité dans des environnements préservés. In : Numéro spécial, L'élevage en Europe : une diversité de services et d'impacts. Dumont B. (Éd). INRA Prod. Anim., 30, 333-350.

Westhoek H., Lesschen J.P., Leip A., Rood T., Wagner S., De Marco A., Murphy-Bokern D., Pallière C., Howard C.M., Oenema O., 2015. Nitrogen on the table: the influence of food choices on nitrogen emissions and the European environment. NERC/Centre for Ecology \& Hydrol., Edinburgh, 66p.

Zabel F., Putzenlechner B., Mauser W., 2014. Global Agricultural Land Resources - A High Resolution Suitability Evaluation and Its Perspectives until 2100 under Climate Change Conditions. Plos One, 9, e107522.

Zhang W., Ricketts T. H., Kremen C., Carney K., Swinton S. M. 2007. Ecosystem services and dis-services to agriculture. Ecol. Econom., 64, 253-260. http://doi.org/10.1016/i.ecolecon. 2007.02 .024

\section{Résumé}

L'élevage et les produits animaux sont souvent examinés par domaine (environnement, économie) ou par niveau d'organisation (exploitation agricole, filière, territoire, pays) si bien qu'il est difficile d'évaluer simultanément la diversité des services et impacts issus de l'élevage dans les territoires. En cohérence avec la littérature scientifique sur les systèmes socio-écologiques, nous avons représenté l'élevage et ses produits à l'interaction de trois systèmes (écologique, technique et social). Ceci permet d'identifier la diversité des facteurs (interactions biotiques, pratiques, modes d'organisation) à l'origine des impacts et des services rendus par l'élevage. Nous en avons ensuite dérivé une représentation simplifiée, «la grange » qui permet de visualiser l'élevage (l'usage des terres, les animaux...) et ses impacts ou services sur cinq interfaces (les intrants, l'environnement et le climat, les marchés, le travail et l'emploi, et les enjeux sociaux et culturels), et les synergies/antagonismes entre services au sein d'un territoire. Nous définissons ensuite différentes formes d'élevage afin de représenter la diversité de l'élevage entre territoires, et d'identifier différentes voies d'innovation pour augmenter ses services et réduire ses impacts. Celles-ci se distinguent par l'insertion des élevages dans les systèmes alimentaires (mondialisés vs territorialisés), ainsi que par l'autonomie en intrants pour l'alimentation des animaux. Cette classification est mobilisée pour introduire les différents cas d'études analysés dans ce numéro spécial.

\section{Abstract}

\section{The "barn": a conceptual framework for understanding the services provided by livestock in a territory}

Livestock and livestock products are often examined by discipline (environment, economy) or by organizational level (farm, sector, territory, country), making it difficult to simultaneously assess the diversity of services and impacts generated by livestock farming systems. Based on the scientific literature, we consider livestock farming systems at the interaction between the ecological, technical and social systems. Doing this, we can identify factors (biotic interactions, system management, collective organization) at the origin of their impacts and services. We then propose a simplified representation, the "barn", which visualizes how livestock systems (described by land use, animal density...) interact with their environment along five interfaces (inputs, environment and climate, markets, labor and employment, social and cultural factors). Two key characteristics of livestock farming systems, their integration into food systems (globalized $v s$. territorialized) and the extent to which they rely on exogenous $v$ s. endogenous inputs, allow proposing a grid to analyze sustainability pathways for each type of territory; we use this grid to position the case studies analyzed in this special issue.

DURU M., DONNARS C., RYCHAWY J., THEROND O., DUMONT B., 2017. La « grange » : un cadre conceptuel pour appréhender les bouquets de services rendus par l'élevage dans les territoires. In : Numéro spécial, L'élevage en Europe : une diversité de services et d'impacts. Dumont B. (Éd). INRA Prod. Anim., 30, 273-284. 